\title{
基于满分集空时分组码的非相干空时码 设计
}

郭永亮 ${ }^{*}$, 朱世华

西安交通大学信息与通信工程系, 西安 710049

*E-mail: ylg@mailst.xjtu.edu.cn

收稿日期: 2005-12-04; 接受日期: 2006-10-19

国家自然科学基金(批准号: 60372055)和教育部博士点基金(批准号: 20030698027)资助项目

摘要基于满分集空时分组码，提出了一种可获得满代数分集的非酉非相

关键词

千空时编码方案，通过将其 “等效” 为酉空时码，对该编码的误码性能进行 了优化. 同时，通过充分利用文中编码方案的结构，提出了复杂度较低的分 组广义似然比检验 GLRT(generalized likelihood ratio test)解码算法. 与现有酉 空时码相比，仿真实验表明，此方案虽在信噪比方面略有损失，但其频谱效 率较高, 且 GLRT 分组解码算法能够对复杂度和性能进行较好的折衰.

代数分集增益 非酉空时码

广义似然比检验 成对错误概率 分组 GLRT 解码

MIMO系统中的空时编码技术通过将时间和空间分集结合起来，分别在时域和空域引入 咒余来提高系统的传输性能. 空时编码可分为网格码和分组码两类, 前者性能较好, 但编码设 计比较困难, 解码复杂度很高; 后者由于引入了正交设计, 具有编解码简单的优点, 因而被 3GPP的WCDMA协议采纳. 然而, 这两种编码在解码时都需要信道状态信息(CSI), 这在天线 数较多或信道变化较快时很难满足 ${ }^{[1]}$, 因此, 研究无需信道估计的非相干空时编码具有很大 的实用价值.

文献 [2]指出当各发射天线的发送向量互相正交时, 非相干MIMO系统可达到其容量, 故 称该类编码为西空时码. 文献 [3]通过引入分组循环相关结构, 利用计算机搜索提出了 “系统设 计”方案，其缺点是难以达到全局最优，且解码复杂度很高. 文献 [4]的“代数设计”方案解码复 杂度虽低, 但其码率至多为 $1 / 2$. 如文献 [5]所指出的, 码字的正交性已成为非相干编码事实上 的标准. 然而, 正交性对编码设计而言是一个较强的约束, 况且西空时码也只有当信噪比较高 或信道相干时间所包括的时隙数远大于发射天线数时才能达到信道容量. 故文献 [5]提出了非 酉非相干空时码，由于其对码字没有正交性约束，故编码设计的自由度较大. 文献 [5]尽管给 出了一些该类编码的例子, 但其解码需要采用球形解码算法 ${ }^{[6]}$, 其实际的解码复杂度是一个 
随机变量, 不利于工程实现. 为此, 本文设计了一类非西非相干编码, 其优点是码率较高, 可 获得满代数分集和存在快速分组解码算法, 相对而言是一种较好的设计方法.

\section{MIMO 系统模型}

考虑一个发射与接收天线数分别为 $M$ 和 $N$ 的无线通信系统. 假设 $h_{i j}$ 为发射天线 $i$ 到接收 天线 $j$ 的信道增益, $M N$ 个统计独立的信道增益均服从 $C N(0,1)$, 且其在 $T$ 个时隙内保持不变. 在时隙 $t$, 从发射天线 $i$ 发送信号 $s_{t i}$, 此时接收天线 $j$ 的接收信号 $y_{t j}$ 为

$$
y_{t j}=\sqrt{\rho / M} \sum_{i=1}^{M} h_{i j} s_{t i}+\eta_{t j},
$$

其中 $\eta_{t j}$ 为时隙 $t$ 接收天线 $j$ 的噪声, $T N$ 个噪声统计独立且均服从 $C N(0,1)$. 假设接收端未知 CSI, $T \times M$ 矩阵 $\boldsymbol{S}=\left(s_{t i}\right)_{T \times M}$ 满足平均功率约束条件 $\mathrm{E}\left[\operatorname{tr}\left(\boldsymbol{S}^{H} \boldsymbol{S}\right)\right]=T M$, 每个接收天线上的信 噪比为 $\rho$. 令 $M \times N$ 矩阵 $\boldsymbol{H}=\left(h_{i j}\right)_{M \times N}, T \times N$ 矩阵 $\boldsymbol{Y}=\left(y_{t j}\right)_{T \times N}, T \times N$ 矩阵 $\boldsymbol{W}=\left(\eta_{t j}\right)_{T \times N}$, 将 (1)式表示为矩阵形式

$$
\boldsymbol{Y}=\sqrt{\rho / M} \boldsymbol{S} \boldsymbol{H}+\boldsymbol{W} .
$$

以下称矩阵 $\boldsymbol{H}, \boldsymbol{S}, \boldsymbol{Y}$ 和 $\boldsymbol{W}$ 分别为信道、发射、接收及噪声矩阵.

文献 [5]中非相干码的出发点是: 在无噪声时(即(2)式中的 $W$ 为零矩阵), 非相干码可只依 据接收矩阵 $\boldsymbol{Y}$ 就可准确地识别出发射矩阵. 换言之, 对任意许用码字矩阵 $\boldsymbol{S}_{i}$, 若信道矩阵的 样本值为 $H_{1}$, 由(2) 式得无噪接收矩阵 $\boldsymbol{Y}$ 为 $\boldsymbol{S}_{i} \boldsymbol{H}_{1}$ (为叙述方便, 此处略去 $\boldsymbol{S}_{i} \boldsymbol{H}_{1}$ 前的系数 $\sqrt{\rho / M}$ ), 由于接收端未知CSI, 故接收机可准确识别出发射矩阵 $\boldsymbol{S}_{i}$ 是指对任意的 $\boldsymbol{H}$ 和任意的 许用码字矩阵 $\boldsymbol{S}_{j}\left(\boldsymbol{S}_{j} \neq \boldsymbol{S}_{i}\right)$, 均成立 $\boldsymbol{S}_{i} \boldsymbol{H}_{1} \neq \boldsymbol{S}_{j} \boldsymbol{H}$, 唯一例外的情形是当 $\boldsymbol{H}_{1}=\mathbf{0}_{M \times N}$ 时, 取 $\boldsymbol{H}=\mathbf{0}_{M \times N}$, 可使 $\boldsymbol{S}_{i} \boldsymbol{H}_{1}=\boldsymbol{S}_{j} \boldsymbol{H}=\mathbf{0}_{T \times N}$. 基于上述观点, 对于非相干MIMO系统, 文献 [5]定义了 代数分集增益(algebraic diversity advantage) 为 $r=M-\max \left\{\operatorname{dim}\left(R\left(\boldsymbol{S}_{i}\right) \cap R\left(\boldsymbol{S}_{j}\right)\right) \mid \boldsymbol{S}_{i} \neq \boldsymbol{S}_{j}\right\}$ (参见文 献 [5]中的定义 3.1 及其(11)式, 值得说明的是, 对于本文的信道模型, 每个码字的衰落块数, 即文献 [5]中的 $M$ 值为 1), 其中 $\boldsymbol{S}_{i}$ 和 $\boldsymbol{S}_{j}$ 为任意两个不同的许用码字矩阵, $\operatorname{dim}$ 表示线性空间的 维数, $R(\cdot)$ 表示矩阵的列空间, $\cap$ 表示线性子空间的交. 若 $r=M$, 则称该码可达到满代数分 集增益.

\section{2 基于满分集分组码的非相干编码设计}

文献 [7]基于正交空时分组码构造了酉空时码, 当发射天线数为 2 和 4 时, 其发射矩阵分 别为下式中的 $\phi_{2}$ 和 $\phi_{4}$ : 


$$
\boldsymbol{\phi}_{2}=\left[\begin{array}{cc}
1 & 0 \\
0 & 1 \\
x_{1} & x_{2} \\
-x_{2}^{*} & x_{1}^{*}
\end{array}\right], \quad \boldsymbol{\phi}_{4}=\left[\begin{array}{cccc}
1 & 0 & 0 & 0 \\
0 & 1 & 0 & 0 \\
0 & 0 & 1 & 0 \\
0 & 0 & 0 & 1 \\
x_{1} & x_{2} & x_{3} & 0 \\
-x_{2}^{*} & x_{1}^{*} & 0 & x_{3} \\
x_{3}^{*} & 0 & -x_{1}^{*} & x_{2} \\
0 & x_{3}^{*} & -x_{2}^{*} & -x_{1}
\end{array}\right],
$$

该码可获得满分集且可实现快速极大似然解码, 但其码率分别只有 $1 / 2$ 和 $3 / 8$. 原因有两点: 一方面, 由于酉空时码需满足正交性约束, 故其只能用正交空时分组码来设计, 但当发射天线 数大于 2 时, 正交空时分组码的码率小于 1 , 这必然会导致所设计的西空时码的码率较低, 例 如, 当发射天线数为 4 时, 正交空时分组码的码率为 $3 / 4$, 这导致了(3)式中 $\phi_{4}$ 的码率只有 $3 / 8$; 另一方面, (3)式在单位阵后面只放置了一个正交码块, 这样 $\phi_{2}$ 和 $\phi_{4}$ 中用来传输信息的时隙数 只有帧长的一半, 这必然导致其码率较低. 对(3)式码率低的缺点, 可分别针对上述两个原因 进行改进. 首先, 由于本文设计的是非西编码, 故设计不必受限于正交空时分组码, 可以选用 码率较高的满分集空时分组码来提高非相干编码的码率; 其次, 本文在设计编码时, 在已知矩 阵后面连续放置了若干个分组码码块, 这可有效地提高码率. 故对发射天线数为 $M$ 的 MIMO 系统，构造非西非相干空时码的发射矩阵 $\boldsymbol{\Psi}_{M}$ 为

$$
\boldsymbol{\Psi}_{M}=\lambda\left[\begin{array}{c}
\boldsymbol{B}_{0} \\
\boldsymbol{B}_{1} \\
\vdots \\
\boldsymbol{B}_{k-1}
\end{array}\right],
$$

其中 $\boldsymbol{B}_{0}$ 为任意 $M$ 阶可逆方阵, 其作用相当于(3)式中的单位阵, $k$ 为不小于 2 的正整数, $\left\{\boldsymbol{B}_{i}\right\}_{i=1}^{k}$ 为 $t \times M$ 阶空时分组码码块, $t$ 为码块的时隙数, 对应于发射天线数为 3 和 $4, \boldsymbol{B}_{i}$ 分别 为 $[8]$

$$
\boldsymbol{B}_{i}^{3}=\left[\begin{array}{ccc}
x_{4 i-3} & x_{4 i-2} & x_{4 i-1} \\
-x_{4 i-2}^{*} & x_{4 i-3}^{*} & \gamma x_{4 i}^{*} \\
x_{4 i-1}^{*} & \gamma x_{4 i}^{*} & -x_{4 i-3}^{*} \\
\gamma x_{4 i} & x_{4 i-1}^{*} & -x_{4 i-2}^{*}
\end{array}\right], \quad \boldsymbol{B}_{i}^{4}=\left[\begin{array}{cccc}
x_{4 i-3} & x_{4 i-2} & x_{4 i-1} & \gamma x_{4 i}^{*} \\
-x_{4 i-2}^{*} & x_{4 i-3}^{*} & \gamma x_{4 i}^{*} & -x_{4 i-1} \\
-x_{4 i-1}^{*} & \gamma x_{4 i} & x_{4 i-3}^{*} & x_{4 i-2} \\
\gamma x_{4 i} & x_{4 i-1}^{*} & -x_{4 i-2}^{*} & x_{4 i-3}
\end{array}\right],
$$

$\boldsymbol{B}_{i}$ 的上角标为发射天线数. 对于给定的星座和 $k$ 值, 选取(4)式中常数 $\lambda$ 以满足功率约束条件 $\mathrm{E}\left[\operatorname{tr}\left(\boldsymbol{\Psi}_{M}^{H} \boldsymbol{\Psi}_{M}\right)\right]=T M$. 若 (5) 式的星座集合为 Gauss 整环 $\mathbb{Z}[i]=\{a+b i \mid a, b \in \mathbb{Z}\}$ 的子集且 Diophantos数 ${ }^{[8]} \gamma$ 的选取能够使 $\left\{1, \gamma, \gamma^{2}, \gamma^{3}, \gamma^{4}\right\}$ 在 $\mathbb{Z}[i]$ 上代数独立时, (5)式的空时分组码可获 得满分集 ${ }^{[8]}$, 在本文中总假定其可获得满分集. 当发射天线数为 4 时, 对比(3)和(4)式, 显然后 
者码率得到了显著提高, 一方面, (4)式中的分组码码率都为 1 , 而正交空时分组码的码率只有 $3 / 4$; 此外, 相比于(3)式, (4)式在可逆方阵后面连续放置了 $k-1$ 个码块, 显然其码率较(3)式高.

\section{3 非相干空时码的代数分集增益}

本节分析(4)式编码的代数分集增益, 为叙述方便, 本节中略去(4)式中的常数 $\lambda$, 它只与 信噪比的标定有关, 不会影响最终结论. 由本文第 1 节可知, 为证明(4)式可获得满代数分集, 只需证对任意两个不同的发射矩阵 $\boldsymbol{S}_{i}$ 和 $\boldsymbol{S}_{j}, \operatorname{dim}\left(R\left(\boldsymbol{S}_{i}\right) \cap R\left(\boldsymbol{S}_{j}\right)\right)=0$ 成立. 利用线性空间的维 数公式 [9], 可将证明目标转化为

$$
\operatorname{dim}\left(R\left(\boldsymbol{S}_{i}\right)+R\left(\boldsymbol{S}_{j}\right)\right)=\operatorname{dim}\left(R\left(\boldsymbol{S}_{i}\right)\right)+\operatorname{dim}\left(R\left(\boldsymbol{S}_{j}\right)\right) .
$$

对上式中的左右两边分别使用恒等式 $\operatorname{dim}\left(R\left(\boldsymbol{S}_{i}\right)+R\left(\boldsymbol{S}_{j}\right)\right)=\operatorname{rank}\left(\left[\boldsymbol{S}_{i} \vdots \boldsymbol{S}_{j}\right]\right)$ 和 $\operatorname{dim}\left(R\left(\boldsymbol{S}_{i}\right)\right)=\operatorname{rank}\left(\boldsymbol{S}_{i}\right)^{[9]}$ 可得

$$
\operatorname{rank}\left(\left[\boldsymbol{S}_{i}: \boldsymbol{S}_{j}\right]\right)=\operatorname{rank}\left(\boldsymbol{S}_{i}\right)+\operatorname{rank}\left(\boldsymbol{S}_{j}\right) .
$$

据(4)式可设 $\boldsymbol{S}_{i}$ 和 $\boldsymbol{S}_{j}$ 分别为

$$
\boldsymbol{S}_{i}=\left[\begin{array}{c}
\boldsymbol{B}_{0} \\
\boldsymbol{B}_{1}^{i} \\
\vdots \\
\boldsymbol{B}_{k-1}^{i}
\end{array}\right], \quad S_{j}=\left[\begin{array}{c}
\boldsymbol{B}_{0} \\
\boldsymbol{B}_{1}^{j} \\
\vdots \\
\boldsymbol{B}_{k-1}^{j}
\end{array}\right],
$$

上式中 $\boldsymbol{B}_{0}$ 为可逆方阵, 故 $\operatorname{rank}\left(\boldsymbol{S}_{i}\right)=M$, 同理 $\operatorname{rank}\left(\boldsymbol{S}_{j}\right)=M$, 此时(7)式为

$$
\operatorname{rank}\left(\left[\boldsymbol{S}_{i} \vdots \boldsymbol{S}_{j}\right]\right)=2 M \text {. }
$$

由(8)式可得

$$
\left[\boldsymbol{S}_{i}: \boldsymbol{S}_{j}\right]=\left[\begin{array}{cc}
\boldsymbol{B}_{0} & \boldsymbol{B}_{0} \\
\boldsymbol{B}_{1}^{i} & \boldsymbol{B}_{1}^{j} \\
\vdots & \vdots \\
\boldsymbol{B}_{k-1}^{i} & \boldsymbol{B}_{k-1}^{j}
\end{array}\right]
$$

对其进行块初等列变换得

$$
\left[\boldsymbol{S}_{i} \vdots \boldsymbol{S}_{j}\right] \rightarrow\left[\boldsymbol{S}_{i} \vdots \boldsymbol{S}_{j}-\boldsymbol{S}_{i}\right]=\left[\begin{array}{cc}
\boldsymbol{B}_{0} & \mathbf{0} \\
\boldsymbol{B}_{1}^{i} & \boldsymbol{B}_{1}^{j}-\boldsymbol{B}_{1}^{i} \\
\vdots & \vdots \\
\boldsymbol{B}_{k-1}^{i} & \boldsymbol{B}_{k-1}^{j}-\boldsymbol{B}_{k-1}^{i}
\end{array}\right] .
$$

矩阵的块初等变换不会改变矩阵的秩 ${ }^{[9]}$, 故欲证(9)式成立, 只需证

$$
\operatorname{rank}\left(\left[\boldsymbol{S}_{i}: \boldsymbol{S}_{j}-\boldsymbol{S}_{i}\right]\right)=2 M \text {. }
$$

注意到 $\left[\boldsymbol{S}_{i}: \boldsymbol{S}_{j}-\boldsymbol{S}_{i}\right]$ 的列数为 $2 M$, 故只需证 $\left[\boldsymbol{S}_{i}: \boldsymbol{S}_{j}-\boldsymbol{S}_{i}\right]$ 存在一个秩为 $2 M$ 的子阵即可, 以下 给出该子阵的构造步骤: 由 $\boldsymbol{S}_{i} \neq \boldsymbol{S}_{j}$ 可知, 存在正整数 $p(1 \leqslant p \leqslant k-1)$, 使得 $\boldsymbol{B}_{p}^{i} \neq \boldsymbol{B}_{p}^{j}$, 此时可 
以证明 $\left[\boldsymbol{S}_{i}: \boldsymbol{S}_{j}-\boldsymbol{S}_{i}\right]$ 的子阵 $\left[\begin{array}{cc}\boldsymbol{B}_{0} & \mathbf{0} \\ \boldsymbol{B}_{p}^{i} & \boldsymbol{B}_{p}^{j}-\boldsymbol{B}_{p}^{i}\end{array}\right]$ 为列满秩阵, 证明如下:

设 $\boldsymbol{B}_{0}, \boldsymbol{B}_{p}^{i}$ 和 $\boldsymbol{B}_{p}^{j}-\boldsymbol{B}_{p}^{i}$ 的列向量组分别为 $\left\{\boldsymbol{x}_{m}\right\}_{m=1}^{M},\left\{\boldsymbol{y}_{m}\right\}_{m=1}^{M}$ 和 $\left\{\boldsymbol{z}_{m}\right\}_{m=1}^{M}$, 则

$$
\left[\begin{array}{cc}
\boldsymbol{B}_{0} & \mathbf{0} \\
\boldsymbol{B}_{p}^{i} & \boldsymbol{B}_{p}^{j}-\boldsymbol{B}_{p}^{i}
\end{array}\right]=\left[\begin{array}{cccccc}
\boldsymbol{x}_{1} & \cdots & \boldsymbol{x}_{M} & \mathbf{0} & \cdots & \mathbf{0} \\
\boldsymbol{y}_{1} & \cdots & \boldsymbol{y}_{M} & \boldsymbol{z}_{1} & \cdots & \mathbf{z}_{M}
\end{array}\right] .
$$

令 $\left[\begin{array}{cc}\boldsymbol{B}_{0} & \mathbf{0} \\ \boldsymbol{B}_{p}^{i} & \boldsymbol{B}_{p}^{j}-\boldsymbol{B}_{p}^{i}\end{array}\right]$ 的 $2 M$ 个列向量的线性组合为零, 可得

$$
k_{1}\left(\begin{array}{l}
\boldsymbol{x}_{1} \\
\boldsymbol{y}_{1}
\end{array}\right)+\cdots+k_{M}\left(\begin{array}{c}
\boldsymbol{x}_{M} \\
\boldsymbol{y}_{M}
\end{array}\right)+k_{M+1}\left(\begin{array}{c}
\mathbf{0} \\
\mathbf{z}_{1}
\end{array}\right)+\cdots+k_{2 M}\left(\begin{array}{c}
\mathbf{0} \\
\mathbf{z}_{M}
\end{array}\right)=\mathbf{0} .
$$

上式可写为

$$
\left\{\begin{array}{l}
k_{1} \boldsymbol{x}_{1}+\cdots+k_{M} \boldsymbol{x}_{M}=0 \\
k_{1} \boldsymbol{y}_{1}+\cdots+k_{M} \boldsymbol{y}_{M}+k_{M+1} \mathbf{z}_{1}+\cdots+k_{2 M} \mathbf{z}_{M}=0 .
\end{array}\right.
$$

由 $B_{0}$ 为可逆方阵可知 $\left\{\boldsymbol{x}_{m}\right\}_{m=1}^{M}$ 线性无关, 故上式中 $k_{1}=\cdots=k_{M}=0$, 此时上式变为

$$
k_{M+1} \mathbf{z}_{1}+\cdots+k_{2 M} \mathbf{z}_{M}=0 .
$$

回顾(4)和(5)及(8)式, 考虑到 $\boldsymbol{B}_{p}^{i}$ 和 $\boldsymbol{B}_{p}^{j}$ 为满分集空时分组码的两个不同的码字矩阵, 由空时码 的秩准则可知 $\boldsymbol{B}_{p}^{j}-\boldsymbol{B}_{p}^{i}$ 列满秩 ${ }^{[10]}$, 即 $\left\{\boldsymbol{z}_{m}\right\}_{m=1}^{M}$ 线性无关, 故由(15)式可得 $k_{M+1}=\cdots=k_{2 M}=0$. 至此可得 (13)式中的 $2 M$ 个组合系数全为零, 故 $\left[\begin{array}{cc}\boldsymbol{B}_{0} & \mathbf{0} \\ \boldsymbol{B}_{p}^{i} & \boldsymbol{B}_{p}^{j}-\boldsymbol{B}_{p}^{i}\end{array}\right]$ 列满秩, 进一步可得 $\left[S_{i}: S_{j}-S_{i}\right]$ 列满秩. 至此可知(11)式成立, 即本文所设计的非相干空时码可获得满代数分集, 这从理论上保证了该编码具有较好的误码性能.

\section{4 非相干空时码的解码算法}

非相干空时码的最大似然解码可表示为

$$
\hat{\boldsymbol{S}}=\arg \max _{\boldsymbol{S} \in \Theta} g(\boldsymbol{Y} \mid \boldsymbol{S}),
$$

其中 $\Theta$ 为所有发射矩阵组成的集合, $g(\boldsymbol{Y} \mid \boldsymbol{S})$ 表示发射矩阵为 $\boldsymbol{S}$ 时接收矩阵 $\boldsymbol{Y}$ 的条件概率密 度, 其表达式为 ${ }^{[1]}$

$$
g(\boldsymbol{Y} \mid \boldsymbol{S})=\frac{\exp \left[-\operatorname{tr}\left(\left[\boldsymbol{I}_{T}+(\rho T / M) \boldsymbol{S} \boldsymbol{S}^{H}\right]^{-1} \mathbf{Y} \mathbf{Y}^{H}\right)\right]}{\pi^{T N}\left|\boldsymbol{I}_{T}+(\rho T / M) S \boldsymbol{S}^{H}\right|^{N}} .
$$

对于本文编码, 若直接使用(17)式解码, 由于涉及到矩阵求逆和行列式计算, 复杂度会很高. 另外, (17)式涉及到信噪比 $\rho$, 故需接收机解码时实时估计 $\rho$ 值, 这会增加系统开销. 鉴于此, 以下考察 GLRT 解码算法. 
GLRT 解码器是对 CSI进行估计后再进行 “相干”解码的 [11]. 若发射矩阵为 $\boldsymbol{S}$, 利用 $\boldsymbol{Y}=\sqrt{\rho / M} \boldsymbol{S} \boldsymbol{H}+\boldsymbol{W}$ 可得 $\boldsymbol{H}$ 的极大似然估计为 $\hat{\boldsymbol{H}}=\sqrt{M / \rho} \boldsymbol{S}^{+} \boldsymbol{Y}$, 其中 $\boldsymbol{S}^{+}$为 $\boldsymbol{S}$ 的伪逆, GLRT解 码准则可表示为

$$
\hat{\boldsymbol{S}}=\arg \min _{\boldsymbol{S} \in \Theta}\|\boldsymbol{Y}-\sqrt{\rho / M} \boldsymbol{S} \hat{\boldsymbol{H}}\|^{2}=\arg \max _{\boldsymbol{S} \in \Theta} \operatorname{tr}\left[\boldsymbol{S}\left(\boldsymbol{S}^{H} \boldsymbol{S}\right)^{-1} \boldsymbol{S}^{H} \mathbf{Y} \boldsymbol{Y}^{H}\right] .
$$

值得指出的是, 对于西空时码, 极大似然解码和GLRT解码是等价的 ${ }^{[3]}$, 两者可表示为

$$
\hat{\boldsymbol{S}}=\arg \max _{\boldsymbol{S} \in \Theta} \operatorname{tr}\left(\boldsymbol{S} \boldsymbol{S}^{H} \mathbf{Y} \boldsymbol{Y}^{H}\right) .
$$

但对于本文的非酉空时码, 对比(17)和(18)式, GLRT 算法的复杂度明显较低, 故本文选用其来 解码.

\section{5 非相干空时码的性能分析}

以(19)式作为解码准则, 文献 [1]中的(5)式给出了酉空时码的成对错误概率PEP(pair-wise error probability)的Cheroff上界为

$$
P_{e}\left(\boldsymbol{S}_{i} \rightarrow \boldsymbol{S}_{j} \mid \boldsymbol{S}_{i}\right) \leqslant \frac{1}{2} \prod_{m=1}^{M}\left\{1+\left(\frac{\rho T}{M}\right)^{2}\left(1-d_{m}^{2}\right) /\left[4\left(1+\frac{\rho T}{M}\right)\right]\right\}^{-N},
$$

其中 $\boldsymbol{S}_{i}$ 和 $\boldsymbol{S}_{j}$ 为任意两个不同的西空时码码字, $\left\{d_{m}\right\}_{m=1}^{M}$ 为矩阵 $\boldsymbol{S}_{i}^{H} \boldsymbol{S}_{j}$ 的奇异值, $P_{e}\left(S_{i} \rightarrow S_{j} \mid S_{i}\right)$ 为发送 $S_{i}$ 但错判为 $S_{j}$ 的概率. 如前节所述, 对于西空时码, 极大似然和 GLRT 解码是等价的, 故(20)式对两个解码准则均有效.

对(4)式的非西空时码, 利用 QR 分解和(20)式可得其 GLRT 解码器的 PEP 上界. 将(18)式 中的 $\boldsymbol{S}$ 进行 $\mathrm{QR}$ 分解可得

$$
\boldsymbol{S}=\boldsymbol{Q}_{S} \boldsymbol{R}_{S},
$$

其中 $T \times M$ 矩阵 $\boldsymbol{Q}_{S}$ 满足 $\boldsymbol{Q}_{S}^{H} \boldsymbol{Q}_{S}=\boldsymbol{I}_{M}, \boldsymbol{R}_{S}$ 为 $M$ 阶上三角阵, 由 $\boldsymbol{S}$ 列满秩可知 $\boldsymbol{R}_{S}$ 可逆 ${ }^{[9]}$. 将(21) 式代入(18)式可得

$$
\hat{\boldsymbol{S}}=\arg \max _{\boldsymbol{S} \in \Theta} \operatorname{tr}\left(\boldsymbol{Q}_{S} \boldsymbol{Q}_{S}^{H} \mathbf{Y} \boldsymbol{Y}^{H}\right) .
$$

巧合的是, (22)和(19)式具有相同的形式, 换言之, 通过 QR 分解可将非西空时码的 GLRT 解码 转化为(19)式所示的解码. 由于 $\boldsymbol{Q}_{S}^{H} \boldsymbol{Q}_{S}=\boldsymbol{I}_{M}$, 故可将 $\boldsymbol{Q}_{S}$ 看作西空时码的一个码字, 利用(20)式 可得 GLRT 解码器的 PEP 上界为

$$
P_{e}\left(S_{i} \rightarrow S_{j} \mid S_{i}\right) \leqslant \frac{1}{2} \prod_{m=1}^{M}\left\{1+\left(\frac{\rho T}{M}\right)^{2}\left(1-d_{m}^{2}\right) /\left[4\left(1+\frac{\rho T}{M}\right)\right]\right\}^{-N} .
$$

基于(21)式可知, 对上式中的码字 $\boldsymbol{S}_{i}$ 和 $\boldsymbol{S}_{j}$, 分别有 $\boldsymbol{S}_{i}=\boldsymbol{Q}_{i} \boldsymbol{R}_{i}$ 和 $\boldsymbol{S}_{j}=\boldsymbol{Q}_{j} \boldsymbol{R}_{j},\left\{d_{m}\right\}_{m=1}^{M}$ 为矩阵 $\boldsymbol{Q}_{i}^{H} \boldsymbol{Q}_{j}$ 的奇异值.

对(4)式中的参数 $\gamma$, 前文只要求其取值能保证(5)式的分组码可获得满分集, 以保证所设 
计的非相干编码可获得满代数分集, 由于满足该条件的 $\gamma$ 值不唯一, 故可选取最优的 $\gamma$ 值对编 码性能进行优化. 由于(23)式的Cheroff界依赖于信噪比 $\rho$, 这会使得最优 $\gamma$ 值的选取比较困难. 为便于优化, 可使用分集积 $\xi_{i, j}=\prod_{m=1}^{M}\left(1-d_{m}^{2}\right)^{1 / 2 M}$ 作为误码性能的替代指标 ${ }^{[4]}$, 该值越大, PEP越小. 分集积是对两个特定的码字而言的, 故对编码的整体性能进行优化可描述为

$$
\gamma_{\mathrm{opt}}=\arg \max _{\gamma \in \Phi} \min _{\mathbf{S}_{i}, \mathbf{s}_{j} \in \Theta(\gamma)} \xi_{i, j},
$$

即 $\gamma$ 的最优值 $\gamma_{\mathrm{opt}}$ 能够最大化最小分集积, 上式中 $\Phi$ 表示参数 $\gamma$ 的取值区域, $\Theta(\gamma)$ 表示(4)式 所示编码的全体发射矩阵所组成的码字集合, $\Theta(\gamma)$ 中的变量 $\gamma$ 表示(4)式的编码依赖于 $\gamma$, $\xi_{i, j}$ 为 $\boldsymbol{S}_{i}\left(Q_{i}\right)$ 和 $\boldsymbol{S}_{j}\left(\boldsymbol{Q}_{j}\right)$ 的分集积. 对(24)式很难求出其解析解, 故采用计算机搜索的方法来逼 近最佳解. 搜索时为了对复杂度和精度进行折衷, 可先选取较大搜索步长, 然后逐渐缩小步长 以得到精度较高的数值解.

值得注意的是, 对非相干空时码, $\xi_{\min }>0$ 与满代数分集等价. 其理由在于: 一方面, 由文 献 [7]中的引理 1 可知 $\operatorname{dim}\left(R\left(\boldsymbol{Q}_{i}\right) \cap R\left(\boldsymbol{Q}_{j}\right)\right)=0$ 与矩阵 $\boldsymbol{Q}_{i}^{H} \boldsymbol{Q}_{j}$ 的最大奇异值 $d_{M}$ 小于 1 等价; 另一 方面, 由 $\xi_{i, j}=\prod_{m=1}^{M}\left(1-d_{m}^{2}\right)^{1 / 2 M}$ 和 $0 \leqslant d_{1} \leqslant \cdots d_{M} \leqslant 1$ 可知, $d_{M}<1$ 和 $\xi_{i, j}>0$ 是等价的, 故 $\operatorname{dim}\left(R\left(\boldsymbol{Q}_{i}\right) \cap R\left(\boldsymbol{Q}_{j}\right)\right)=0$ 与 $\xi_{i, j}>0$ 是等价的. 此外考虑到 $\boldsymbol{S}_{i}=\boldsymbol{Q}_{i} \boldsymbol{R}_{i}$ 和 $\boldsymbol{S}_{j}=\boldsymbol{Q}_{j} \boldsymbol{R}_{j}$, 且 $\boldsymbol{R}_{i}$ 和 $\boldsymbol{R}_{j}$ 均 为可逆方阵, 故有

$$
R\left(\boldsymbol{Q}_{i}\right)=R\left(\boldsymbol{S}_{i}\right), \quad R\left(\boldsymbol{Q}_{j}\right)=R\left(\boldsymbol{S}_{j}\right) .
$$

至此可知 $\operatorname{dim}\left(R\left(\boldsymbol{Q}_{i}\right) \cap R\left(\boldsymbol{Q}_{j}\right)\right)=\operatorname{dim}\left(R\left(\boldsymbol{S}_{i}\right) \cap R\left(\boldsymbol{S}_{j}\right)\right)=0$ 等价于 $\xi_{i, j}>0$, 由代数分集增益的定义 可知 $\xi_{\text {min }}>0$ 时本文编码可获得满代数分集.

\section{6 仿真结果}

本节对本文的编码方案进行性能仿真, 比较对象为文献 [3]中的西空时码, 两个编码的发 射和接收天线数分别为 3 和 1 , 对本文方案, 选取(4)式中的参数 $k=2$, 调制方式为 Gray映射的 QPSK, 其谱效率为 8/7 bps $/ \mathrm{Hz}$, 采用 GLRT解码器, 选取(4)式中的 $\boldsymbol{B}_{0}$ 为 $\boldsymbol{I}_{3}$. 为了降低发射机 的峰均比, 本文将参数 $\gamma$ 限定在复平面的单位圆上, 即 $|\gamma|=1$. 通过以步长 $2 \pi / 500$ 在区间 $[0,2 \pi)$ 上依据(24)式进行搜索, 可得 $\gamma$ 的最佳取值 $\gamma_{o p t}$ 为 $\mathrm{e}^{281 \pi i / 250}$, 其所对应最小分集积 $\xi_{\text {min }}$ 为 $2.58 \times 10^{-6}$, 注意到 $\xi_{\text {min }}>0$, 故该编码可实现满代数分集. 比较对象的帧长 $T=8$, 频谱效率 为 $1 \mathrm{bps} / \mathrm{Hz}$, 其具体的编码参数和误码曲线可参见文献 [3].

图 1 为误比特率的比较曲线, 本文和文献 [3]中的方案分别标识为“非酉设计”和“系统设 计”. 如图 1 所示, 前者相对后者虽约有 $1 \mathrm{~dB}$ 的信噪比损失, 但前者的谱效率比后者提高了约 $14 \%$. 比较“非西设计”和“系统设计”的解码复杂度, 后者属于西空时码, 其极大似然解码如 (19)式所示，对“非西设计”, 由前述分析，其GLRT解码可采用(22)式实现，显然两个解码具有 
相同的形式. 为了减少解码的计算复杂度, 预先在接收端存储(19)式中的 $\boldsymbol{S} \boldsymbol{S}^{H}$ ((22)式中的 $\left.\boldsymbol{Q}_{S} \boldsymbol{Q}_{S}^{H}\right)$, 由于两者需存储的矩阵个数相同 $\left(256\right.$ 个), 但 $\boldsymbol{S} \boldsymbol{S}^{H}$ 的维数为 $8 \times 8, \boldsymbol{Q}_{S} \boldsymbol{Q}_{S}^{H}$ 的维数为 $7 \times 7$, 故前者的存储空间为后者的 64/49 倍. 同理可知, 由于 $\boldsymbol{Q}_{S} \boldsymbol{Q}_{S}^{H}$ 的维数较 $\boldsymbol{S} \boldsymbol{S}^{H}$ 低, 故“系 统设计”所需的乘法次数为“非西设计”的 64/49 倍. 另外, 由于“非西设计”只要求信道在 7 个时 隙内保持不变, 而“系统设计”却要求信道在 8 个时隙内保持不变, 故前者在适应信道的时变性 方面有略微优势.

从(4)式可知, 当参数 $k$ 较大时, 码字矩阵的维数会增大, 解码的空间和时间复杂度必然也 会增大. 鉴于此, 本文针对(4)式的编码提出了一种次优算法, 为叙述方便, 下面以 $M=3$, $k=3$ 为例进行说明. 设发射和接收矩阵分别为 $\left[\begin{array}{lll}\boldsymbol{I}_{3} & \boldsymbol{B}_{1}^{\mathrm{T}} & \boldsymbol{B}_{2}^{\mathrm{T}}\end{array}\right]^{\mathrm{T}}$ 和 $\left[\boldsymbol{R}_{0}^{\mathrm{T}} \boldsymbol{R}_{1}^{\mathrm{T}} \boldsymbol{R}_{2}^{\mathrm{T}}\right]^{\mathrm{T}}$, 其中 $\boldsymbol{R}_{0}, \boldsymbol{R}_{1}$, $R_{2}$ 分别为对应于 $I_{3}, B_{1}, B_{2}$ 的接收数据. 考虑到 $B_{1}$ 和 $B_{2}$ 是独立编码的, 一种可行的办法是 依次使用 $\left[\boldsymbol{R}_{0}^{\mathrm{T}} \boldsymbol{R}_{1}^{\mathrm{T}}\right]^{\mathrm{T}}$ 和 $\left[\boldsymbol{R}_{0}^{\mathrm{T}} \boldsymbol{R}_{2}^{\mathrm{T}}\right]^{\mathrm{T}}$ 及(22)式分别解得 $\boldsymbol{B}_{1}$ 和 $\boldsymbol{B}_{2}$ 中的未知符号, 由于其解码是一组 一组完成的, 故称其为分组解码. 图 2 对分组解码进行了仿真, 接收天线数为 2 , 参数 $k=3$, 其他参数和图 1 相同, 显然此时频谱效率为 $16 / 11 \mathrm{bps} / \mathrm{Hz}$. 如图 2 所示, 分组解码与 GLRT 解码 相比, 两者信噪比相差不到 $1 \mathrm{~dB}$. 差异存在的原因是: 接收端解码时无需信道估计, 尽管 $\boldsymbol{B}_{1}$ 和 $\boldsymbol{B}_{2}$ 是互相独立编码的, 但其所对应的接收数据 $\boldsymbol{R}_{1}$ 和 $\boldsymbol{R}_{2}$ 却通过信道矩阵产生了间接的联系, 即接收数据间是相关的, 分组解码正是由于忽略了接收数据内部的相关性, “武断地”认为接收 数据间互相独立而导致了其与 GLRT 解码相比有 $1 \mathrm{~dB}$ 的性能差异.

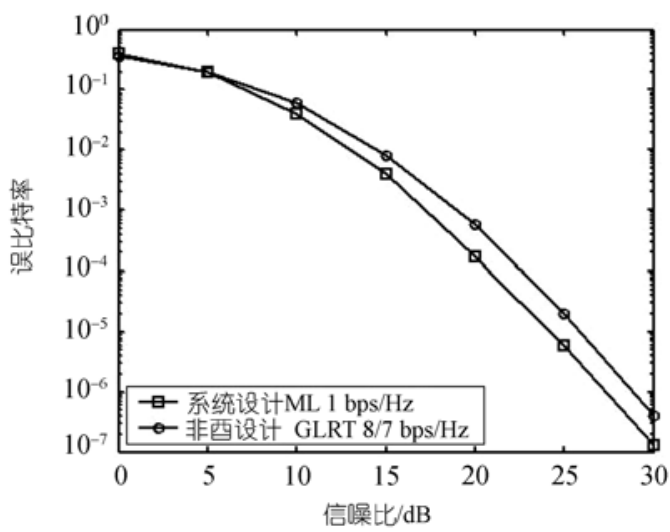

图 1 非酉设计与系统设计误码率比较曲线

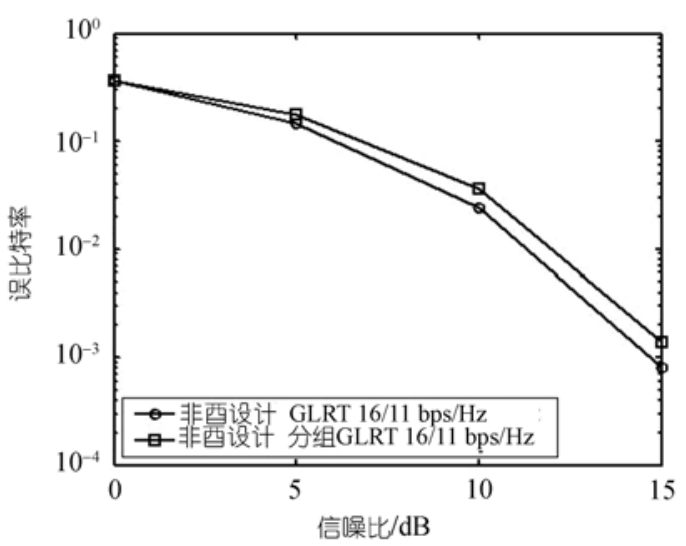

图 2 分组解码与 GLRT 解码误码率比较曲线

通过计算可知, 对应于图 2, GLRT 解码的时间(只计乘法次数)和空间复杂度分别为分组解 码的 316 和 632 倍. 通过估算可知, GLRT 解码的时间(只计算乘法次数)和空间复杂度分别约为 分组算法相应量的 $g^{4 k-8} k / 3$ 和 $g^{4 k-8} k^{2} / 3$ 倍, 其中 $g$ 为调制星座的点数. 所以, 无论是时间还 是空间复杂度, 分组解码能够对复杂度和性能进行较好的折衷. 显然, 分组解码相对于 GLRT 解码还具有解码延时小的优点. 
在本文即将结束时, 有一点值得指出: 表面上看, 可增加(4)式中的 $k$ 值来获取更高的码 率, 但这要求信道具有较大的相关时间, 从这点上讲, 本文方案只是实现了在一定的信道相关 时间下, 选取尽可能大的 $k$ 值来获得较高的码率, 即本文方案能够充分地利用信道的相干时间 获得较高的码率，实现了码率与信道相干时间的最佳匹配.

\section{7 结论}

本文提出了一种基于满分集空时分组码的非西非相干空时编码方案, 该方案可获得满代 数分集并具有较高的码率. 同时, 针对 GLRT 解码器复杂度过高的缺点, 通过充分利用本文方 案的特点, 提出了较实用的分组解码算法. 仿真结果表明, 与 GLRT 算法比较, 分组解码能够 对复杂度和性能损失进行较好的折衰.

\section{参考文献}

1 Marzetta T L, Hochwald B M. Unitary space-time modulation for multiple-antenna communications in Rayleigh flat fading. IEEE Trans Inf Theory, 2000, 46(2): 543-564 [DOI]

2 Marzetta T L, Hochwald B M. Capacity of mobile multiple-antenna communication link in Rayleigh flat fading. IEEE Trans Inf Theory, 1999, 45(1): 139-157 [DOI]

3 Hochwald B M, Marzetta T L, Richardson T J, et al. Systematic design of unitary space-time constellations. IEEE Trans Inf Theory, 2000, 46(6): 1962-1973 $\underline{\text { [DOI] }}$

4 Zhao W L, Leus G, Giannakis. Algebraic design of unitary space-time constellations. IEEE International Conferen Commun, 2003, 5: 3180-3184

5 Gamal H E, Aktas D, Damen M O. Noncoherent space time coding: an algebraic perspective. IEEE Trans Inf Theory, 2005, 51(7): 2380-2390 [DOI]

6 Damen O, Chkeif A, Belfiore J C. Lattice code decoder for space-time codes. IEEE Commun Lett, 2000, 4(5): $161-163 \underline{\text { [DOI] }}$

7 Tarokh V, Kim I M. Existence and construction of noncoherent unitary space-time codes. IEEE Trans Inf Theory, 2002, 48(12): 3112-3117 [DOI]

8 Damen M O, Gamal H E, Beaulieu N C. On the design of quasi-orthogonal constellations: filling the empty threads. IEEE Vehicular Technology Conference, 2003, 3: 1753-1756

9 Horn R A, Johnson C R. Matrix Analysis. Cambridge: Cambridge University Press, 1985. 400-401

10 Tarokh V, Seshadri N, Calderbank A R. Space-time codes for high data wireless communications: performance criterion and code construction. IEEE Trans Inf Theory, 1998, 44(3): 744-765 [DOI]

11 Kay S M. Fundamentals of Statistical Signal Processing-Estimation Theory. Englewood Cliffs: Prentice-Hall, 1993. 203-207 